\title{
O padrão de uso de drogas por grupos em diferentes fases de tratamento nos Centros de Atenção Psicossocial Álcool e Drogas (CAPS-AD)
}

\author{
Drug use of groups in distinct phases of treatment in \\ Psychosocial Care Centers for Alcohol and Drug (CAPS-AD) \\ Daniele do Rocio Ribeiro', Denise Siqueira de Carvalho ${ }^{2}$
}

\section{RESUMO}

Objetivo: Analisar as características sociodemográficas e o padrão de uso de drogas em pacientes dos CAPS-AD de Curitiba, Paraná. Métodos: Trata-se de um estudo observacional, transversal e descritivo em uma amostra de 268 indivíduos, 184 em início e 84 no terceiro mês de tratamento. Os questionários foram aplicados no período entre abril e setembro de 2012 e, além das variáveis sociodemográficas pesquisadas, obtiveram-se informações sobre quais substâncias eram utilizadas, tempo e frequência de uso. Para análise dos dados, utilizaram-se os programas Excel e Epi-Info. Médias e testes de qui-quadrado foram utilizados para a comparação dos grupos. Resultados: Em ambos os grupos, predominaram sexo masculino, baixa escolaridade, baixa renda, ser da cor branca, residir com familiar ou amigo e possuir moradia própria. As drogas lícitas foram as mais utilizadas, e a maconha foi a droga ilícita mais utilizada na vida. O crack foi a droga mais utilizada no último ano e no último mês. Inalantes e alucinógenos foram os menos utilizados. Pacientes em terceiro mês de tratamento

\section{Palavras-chave}

Transtornos relacionados ao uso de substâncias, serviços de saúde mental, usuários de drogas. eram significativamente mais velhos, casados, tiveram menor uso na vida e no último mês de maconha, cocaína e crack, faziam uso mais frequente de álcool, e mais de um terço nunca tinha usado droga ilícita na vida. Conclusão: Os achados deste estudo podem subsidiar o desenvolvimento de estratégias que possibilitem que grupos vulneráveis possam acessar e se manter em tratamento nos CAPS-AD.

\section{ABSTRACT}

Objective: The purpose of the study was to analyze the socio-demographic characteristics and the pattern of drug use in patients of CAPS-AD in Curitiba, Paraná. Methods: A descriptive cross-sectional study was carried out in a sample of 268 individuals, of which 184 in early phase and 84 in the third month of treatment. Between April and September of 2012, the data was collected with the application of a questionnaire that contained sociodemographic questions and information about characteristics of drug use as substances, time and frequency of use. For data analysis, the programs of Excel and Epi-Info were used. Averages and chi-square tests were calculated in order to compare the two groups. Results: The predominant characteristics in both groups were: male, white color, low education, low income,

1 Universidade Federal do Paraná (UFPR).

2 UFPR, Departamento de Saúde Comunitária e Programa de Pós-Graduação em Segurança Alimentar e Nutricional.

Recebido em

$3 / 10 / 2014$

Aprovado em

27/7/2015

DOI: 10.1590/0047-2085000000082

Endereço para correspondência: Daniele do Rocio Ribeiro

Rua Renato Polatti, 3525, ap. 303, bloco 1

81230170 - Curitiba, PR, Brasil

E-mail: dnutri@gmail.com 


\section{Keywords}

Substance-related

disorders, mental health

services, drug users. reside with family or friend and own their house. Legal drugs were the most used. Marijuana was the illicit drug most used in life. Considering the last year and last month, crack was the most used. Inhalants and hallucinogens were the least used. Patients in the third month of treatment were significantly older, married and lifetime use of drugs was lower compared to the other group. Also, use of marijuana, cocaine and crack were lower in the last month. Nonetheless, they made more frequent use of alcohol and, over a third had never used illicit drugs in life. Conclusion: The findings may help to guide new strategies to increase access to treatment of vulnerable groups and also to remain in treatment in the CAPS-AD.

\section{INTRODUÇÃO}

O consumo de drogas no Brasil teve sua prevalência aumentada nos últimos anos. Segundo o Relatório Mundial sobre Drogas 2013, publicado pela Organização das Nações Unidas, enquanto o uso de cocaína em muitos países sul-americanos diminuiu ou se manteve estável, no Brasil houve aumento'. Em 2005, o uso na vida para qualquer droga (exceto tabaco e álcool) era de 22,8\% contra 19,4\% em 20012,3. Esses dados eram semelhantes aos do Chile $(23,4 \%)$ e representavam quase a metade do índice dos Estados Unidos $(45,8 \%)^{4}$. Em relação ao tratamento, a pesquisa realizada em 2005 demonstrou que 2,9\% dos entrevistados haviam recebido tratamento no último ano para abuso de álcool e outras drogas, sendo a maior parcela do sexo masculino e na faixa etária acima de 18 anos $^{2,3}$.

No Brasil, no âmbito do Sistema Único de Saúde (SUS), o tratamento às pessoas com problemas decorrentes do uso ou abuso de álcool e outras drogas tem como referência os Centros de Atenção Psicossocial Álcool e Drogas (CAPS-AD). Os CAPS-AD têm como princípio a prevenção e a promoção à saúde, a interdisciplinaridade, a reinserção social, a atuação na comunidade e a substituição aos antigos manicômios. Também englobam atividades terapêuticas e preventivas, por meio de atendimento individual (medicamentoso, psicoterápico, de orientação), atendimento em grupo (psicoterapia, grupo operativo, atividade de suporte social), oficinas terapêuticas, visitas domiciliares, atendimento às famílias e atividades comunitárias 5 . Atualmente, os CAPS são regulamentados pela Portaria no 336/GM, de 19 de fevereiro de 2002, e integram a rede de atenção à saúde mental do SUS.

Duas pesquisas, realizadas em CAPS-AD em diferentes capitais brasileiras, descreveram o perfil socioeconômico e a adesão ao tratamento dos pacientes. A primeira, realizada em Teresina/PI, fez um levantamento de informações presentes em 227 prontuários ${ }^{6}$. A outra, envolvendo 316 prontuários, foi realizada em Campo Grande/MS. Em ambas, a maioria dos usuários do serviço era do sexo masculino, adulta e com baixa escolaridade. Em Teresina, 55\% faziam uso diário de bebida alcoólica e $29 \%$ faziam uso de drogas ilícitas, sendo a maconha a mais consumida (95,3\%). A permanência em tratamento por pelo menos três meses foi de $43,1 \%^{6}$. Em Campo Grande, predominavam pacientes usuários de álcool, que faziam uso em média de 2,2 tipos de drogas, com média de início de uso aos 17,3 anos de idade e 18,1 anos de uso em média. A maioria nunca fez tratamentos anteriores para deixar o uso abusivo de álcool e outras drogas. Na comparação entre os grupos com maior e menor adesão ao tratamento, utilizando-se o ponto de corte de três meses, havia tendência de maior prevalência de pacientes do sexo masculino, idade mais avançada, registro por pai e mãe e maior tempo de uso de drogas no grupo com mais de três meses de tratamento.

Conhecer as características da população atendida pelos CAPS-AD, tanto daqueles que estão começando o tratamento quanto daqueles que já estão em um período maior de atendimento, gera subsídios para o desenvolvimento de estratégias e ações mais eficazes na atenção aos dependentes de álcool e outras drogas, contemplando as reais necessidades dessa população. Diante disso, esta pesquisa teve por objetivo caracterizar o perfil socioeconômico e demográfico, bem como o padrão de uso de drogas, dos pacientes em início de tratamento e com três meses de tratamento nos CAPS-AD.

\section{MÉTODOS}

Este trabalho é um estudo observacional, transversal e descritivo. Ele foi desenvolvido em todos os cinco CAPS-AD que atendiam adultos na cidade de Curitiba/PR. O fluxo de atendimento nesses serviços tinha como porta de entrada a unidade de saúde ${ }^{8}$. A pesquisa compreendeu dois grupos de pacientes: aqueles que estavam iniciando e outro grupo que estava no terceiro mês de tratamento. Todos os pacientes avaliados possuíam diagnóstico de dependência de alguma droga psicoativa ou de múltiplas drogas.

Para o cálculo amostral, foi utilizado o programa StatCalc do Epi-Info versão 6.04, que estabeleceu uma amostra de 171 indivíduos no grupo em início de tratamento e de 134 no grupo no terceiro mês de tratamento, compreendendo a faixa etária de 18 a 69 anos de idade. Não apresentar condições cognitivas para responder aos questionários, ser gestante ou apresentar deficiência física foram critérios de exclusão.

A coleta de dados foi realizada no período de abril a setembro de 2012, utilizando questionários aplicados pelo mesmo entrevistador. Para a avaliação do perfil socioeconômico e demográfico, foram utilizadas perguntas fechadas sobre idade, sexo, estado civil, raça/cor autorreferidas, renda, ocupação, escolaridade, condições de moradia, número de pessoas por domicílio e participação em programa de transferência de renda. 
As questões relacionadas ao padrão de uso das drogas, tais como substâncias utilizadas, tempo e frequência de uso, foram adaptadas do questionário utilizado pelo Centro Brasileiro de Informações sobre Drogas Psicotrópicas da Universidade Federal de São Paulo no II Levantamento Domiciliar sobre o Uso de Drogas Psicotrópicas no Brasil - $2005^{3}$.

O instrumento de coleta de dados foi previamente testado com um grupo de 10 pacientes em uma das unidades estudadas, objetivando conhecer e adequar problemas na linguagem utilizada e compreensão das perguntas.

Para a apresentação do padrão de uso, utilizaram-se os critérios de classificação de uso de droga propostos pelo Cebrid (2005): uso na vida, quando o indivíduo fez uso da droga pelo menos uma vez na vida; uso no ano, quando fez uso pelo menos uma vez nos últimos 12 meses; uso no mês, quando fez uso da droga pelo menos uma vez nos últimos 30 dias.

Na digitação e análise dos dados, utilizou-se o programa Epi-Info versão 3.5.4. Para as variáveis categóricas, foram calculadas proporções e aplicados os testes de qui-quadrado ou exato de Fisher, e para variáveis contínuas, foram utilizadas as médias e desvios-padrões. Adotou-se o nível de significância inferior a 0,05.

Este estudo foi aprovado pelo Comitê de Ética em Pesquisa do Setor de Ciências da Saúde da Universidade Federal do Paraná, e a tomada de dados junto a cada indivíduo só ocorreu após apresentação e assinatura do Termo de Consentimento Livre e Esclarecido.

\section{RESULTADOS}

Foram avaliados neste estudo 268 pacientes em tratamento nos CAPS-AD em Curitiba/PR, sendo 184 em início de tratamento e 84 pacientes que estavam completando três meses de tratamento. A amostra pretendida de 134 pacientes no terceiro mês não foi alcançada em virtude da inexistência do número de pacientes que obedecessem ao critério de seleção nesse grupo, que era estar completando três meses de tratamento durante a fase de coleta de dados.

O grupo de início de tratamento nesses CAPS-AD era, em sua maioria, composto por homens brancos $(54,3 \%)$, na faixa etária de 31 a 40 anos (32,6\%) e não casados (60,9\%). No entanto, a maioria declarou residir com a família conjugal, isto é, com cônjuge ou filhos (45,1\%). O estrato de renda predominante foi de 0,5 a 1,49 salário mínimo per capita (49,7\%), e a renda familiar média foi de $\mathrm{R} \$ 1.458,62$ (SD $\pm 1.320,60)$. Esses pacientes apresentavam baixa escolaridade: mais da metade não tinha o primeiro grau completo $(51,1 \%)$. Em relação à moradia, a maioria possuía casa própria (56\%) e o tipo de construção que prevaleceu foi de alvenaria (68,5\%). A média de pessoas por domicílio foi de 3,3 (SD $\pm 1,86$ ) e a maior parcela não participava de nenhum programa de transferência de renda $(83,7 \%)$ (Tabela 1).
Tabela 1. Características socioeconômicas e demográficas por grupo de pacientes nos CAPS-AD de Curitiba, Paraná

\begin{tabular}{|c|c|c|c|}
\hline \multirow[t]{2}{*}{ Variável } & $\begin{array}{l}\text { Início de } \\
\text { tratamento }\end{array}$ & $\begin{array}{l}3^{\circ} \text { mês de } \\
\text { tratamento }\end{array}$ & \multirow[t]{2}{*}{$p\left(\chi^{2}\right)$} \\
\hline & $n=184(\%)$ & $n=84(\%)$ & \\
\hline \multicolumn{4}{|l|}{ Faixa etária } \\
\hline Menos que 31 anos & $53(28,8)$ & $10(11,9)$ & $<0,001^{* *}$ \\
\hline 31 a 40 anos & $60(32,6)$ & $21(25,0)$ & \\
\hline 41 a 50 anos & $51(27,7)$ & $33(39,3)$ & \\
\hline Mais que 50 anos & $20(10,9)$ & $20(23,8)$ & \\
\hline \multicolumn{4}{|l|}{ Sexo } \\
\hline Masculino & $147(79,9)$ & $71(84,5)$ & 0,37 \\
\hline Feminino & $37(20,1)$ & $13(15,5)$ & \\
\hline \multicolumn{4}{|l|}{ Estado civil referido } \\
\hline Casado(a)/união estável & $72(39,1)$ & $36(42,9)$ & 0,56 \\
\hline $\begin{array}{l}\text { Não casado(a) (solteiro/ } \\
\text { divorciado/viúvo) }\end{array}$ & $112(60,9)$ & $48(57,1)$ & \\
\hline \multicolumn{4}{|l|}{ Raça/cor autorreferida } \\
\hline Branca & $100(54,3)$ & $53(63,1)$ & ref. \\
\hline Preta & $22(12,0)$ & $4(4,8)$ & $0,05^{*}$ \\
\hline Parda & $60(32,6)$ & $25(29,8)$ & 0,41 \\
\hline Indígena & $2(1,1)$ & $2(2,4)$ & $0,61^{*}$ \\
\hline \multicolumn{4}{|l|}{ Escolaridade } \\
\hline $\begin{array}{l}\text { Ensino fundamental incompleto/ } \\
\text { analfabeto }\end{array}$ & $94(51,1)$ & $44(52,4)$ & $0,94^{* *}$ \\
\hline Ensino fundamental completo & $22(12,0)$ & $9(10,7)$ & \\
\hline Ensino médio incompleto & $16(8,7)$ & $7(8,3)$ & \\
\hline Ensino médio completo & $47(25,5)$ & $20(23,8)$ & \\
\hline $\begin{array}{l}\text { Ensino superior completo/ pós- } \\
\text {-graduação }\end{array}$ & $5(2,7)$ & $4(4,8)$ & \\
\hline \multicolumn{4}{|l|}{ Com quem reside } \\
\hline Só & $25(13,6)$ & $14(16,7)$ & ref. \\
\hline Família conjugal & $83(45,1)$ & $42(50,0)$ & 0,79 \\
\hline Família de origem & $61(33,2)$ & $21(24,0)$ & 0,24 \\
\hline Parentes e amigos & $7(3,8)$ & $4(4,8)$ & $1,00^{*}$ \\
\hline Outro & $8(4,3)$ & $3(3,6)$ & 0,73 \\
\hline \multicolumn{4}{|l|}{$\begin{array}{l}\text { Renda familiar per capita } \\
\text { (salários-mínimos***) }\end{array}$} \\
\hline Menos que 0,5 & $68(37,2)$ & $28(33,3)$ & $0,18^{* *}$ \\
\hline 0,5 a 1,49 & $91(49,7)$ & $39(46,4)$ & \\
\hline 1,5 a 3 & $20(10,9)$ & $14(16,7)$ & \\
\hline Mais que 3 & $4(2,2)$ & $3(3,6)$ & \\
\hline \multicolumn{4}{|l|}{ Tipo de moradia } \\
\hline Casa ou apartamento & $177(96,2)$ & $79(94,0)$ & ref. \\
\hline Abrigo público ou nas ruas & $3(1,6)$ & $1(1,2)$ & $1,00^{*}$ \\
\hline Pensão & $2(1,1)$ & $2(2,4)$ & $0,59^{*}$ \\
\hline Outro & $2(1,1)$ & $2(2,4)$ & $0,59^{*}$ \\
\hline \multicolumn{4}{|l|}{ Situação de moradia } \\
\hline Própria & $103(56,0)$ & $53(63,1)$ & ref. \\
\hline Alugada & $48(26,1)$ & $13(15,5)$ & 0,07 \\
\hline Cedida & $24(13,0)$ & $9(10,7)$ & 0,45 \\
\hline Outra & $9(4,9)$ & $9(10,7)$ & 0,18 \\
\hline \multicolumn{4}{|l|}{ Tipo de construção } \\
\hline Alvenaria & $126(68,5)$ & $59(70,2)$ & ref. \\
\hline Madeira & $33(17,9)$ & $14(16,7)$ & 0,78 \\
\hline Mista & $20(10,9)$ & $8(9,5)$ & 0,73 \\
\hline Outro & $5(2,7)$ & $3(3,6)$ & $0,74^{*}$ \\
\hline \multicolumn{4}{|l|}{$\begin{array}{l}\text { Participa de algum } \\
\text { programa de transferência } \\
\text { de renda }\end{array}$} \\
\hline Sim & $30(16,3)$ & $11(13,1)$ & 0,50 \\
\hline Não & $154(83,7)$ & $73(86,9)$ & \\
\hline
\end{tabular}


O grupo de pacientes em terceiro mês de tratamento, por sua vez, apresentou características socioeconômicas e demográficas semelhantes às do primeiro, com exceção da faixa etária e estado civil: a maioria apresentava-se entre 41 e 50 anos $(39,3 \%)(p<0,05)$ e era casada $(57,1 \%)$, porém sem diferença significativa em relação ao outro grupo (Tabela 1).

Em relação ao padrão de uso de drogas (Tabela 2), o álcool e o tabaco foram as mais utilizadas na vida, no ano e no mês em ambos os grupos de pacientes. A droga ilícita mais utilizada na vida foi a maconha. Quando avaliado o uso no último ano, a droga ilícita mais utilizada foi o crack, assim como quando considerado o uso no último mês. Os inalantes e alucinógenos foram as drogas que apresentaram menor uso, tanto na vida como no ano e no último mês, nos dois grupos de pacientes.

Na comparação entre os dois grupos, verificou-se que no grupo de início maior percentual de entrevistados relatou ter feito uso na vida de maconha, cocaína e crack, com diferença estatisticamente significativa. Em relação ao uso no último ano, não houve diferença estatística para a maioria das drogas, com exceção da cocaína, com maior percentual no grupo de início de tratamento. No entanto, quando avaliado o uso no mês, esse grupo apresentou maior uso das drogas em geral $(p<0,05)$, com exceção apenas dos alucinógenos e do tabaco (Tabela 2).

Verificou-se que a maioria dos entrevistados fez uso de um ou mais tipos de drogas por mais de 10 anos. Álcool, seguido do tabaco, maconha, cocaína e crack foram as utilizadas por mais tempo (mais que 10 anos) em ambos os grupos estudados. Daqueles que afirmaram ter feito uso na vida de alucinógenos, a maioria fez uso experimental, isto é, poucos episódios de uso, de uma a três vezes. Daqueles que afirmaram ter feito uso de inalantes, no grupo de início de tratamento, a maioria utilizou a droga por menos de um ano
- já no outro grupo a maioria fez uso experimental. Também no grupo de início de tratamento, aqueles com tempo de uso de cocaína de dois a cinco anos foi maior, com diferença estatisticamente significativa (Tabela 3).

A respeito da frequência de uso, as drogas usadas com maior frequência semanal (todos os dias) no grupo de início de tratamento, em ordem decrescente, foram: tabaco, álcool, maconha, crack, outras, cocaína, inalantes e alucinógenos. No grupo de terceiro mês, por sua vez, foram: tabaco, álcool, crack, maconha, cocaína, inalantes, outras e alucinógenos. Na comparação entre os dois grupos, houve diferença estatisticamente significativa entre aqueles que faziam uso de álcool de uma a três vezes na semana, com maior percentual de indivíduos no grupo de início. Por outro lado, essa diferença ocorreu também entre aqueles que utilizavam álcool diariamente, porém com maior percentual no grupo de terceiro mês. Daqueles que usavam crack todos os dias, o maior percentual estava entre o grupo de início de tratamento $(p<0,05)$ (Tabela 4).

Entre os pacientes em início de tratamento, 17,9\% revelaram nunca ter feito uso de drogas ilícitas, usando na vida apenas drogas lícitas (como álcool e tabaco). No grupo de terceiro mês, esse percentual sobe para 36,9\%, com diferença significante entre os dois grupos $(p<0,05)$ (Tabela 5).

\section{DISCUSSÃO}

A predominância de homens nos serviços de tratamento para dependência química verificada aqui é uma realidade presente também em outros estudos $5^{6,79-13}$. Levantamentos nacionais sobre o uso de drogas têm mostrado que a taxa de consumo é maior entre os homens ${ }^{2,3}$. O II Levantamento Domiciliar sobre o Uso de Drogas Psicotrópicas no Brasil - 2005

Tabela 2. Padrão de uso de drogas entre os grupos de pacientes estudados nos CAPS-AD de Curitiba, Paraná

\begin{tabular}{|c|c|c|c|c|c|c|c|c|c|}
\hline \multirow{3}{*}{ Drogas } & \multicolumn{3}{|c|}{ Uso na vida } & \multicolumn{3}{|c|}{ Usono ano } & \multicolumn{3}{|c|}{ Usono mês } \\
\hline & $\begin{array}{l}\text { Início } \\
\text { (N 184) }\end{array}$ & $\begin{array}{l}3^{0} \text { mês } \\
\text { (N 84) }\end{array}$ & $p\left(\chi^{2}\right)$ & $\begin{array}{l}\text { Início } \\
\text { (N 184) }\end{array}$ & $\begin{array}{l}3^{\circ} \text { mês } \\
\text { (N 84) }\end{array}$ & $p\left(\chi^{2}\right)$ & $\begin{array}{l}\text { Início } \\
\text { (N 184) }\end{array}$ & $\begin{array}{l}3^{0} \text { mês } \\
\text { (N 84) }\end{array}$ & $p\left(\chi^{2}\right)$ \\
\hline & $n(\%)$ & $n(\%)$ & & $n(\%)$ & $n(\%)$ & & $n(\%)$ & $n(\%)$ & \\
\hline Álcool & $184(100)$ & $84(100)$ & 1,00 & $174(94,6)$ & $79(94,0)$ & 0,86 & $113(61,4)$ & $18(21,4)$ & $<0,000$ \\
\hline Tabaco & $152(82,6)$ & $76(90,5)$ & 0,09 & $132(71,7)$ & $67(79,8)$ & 0,16 & $123(66,8)$ & $62(73,8)$ & 0,25 \\
\hline Maconha & $143(77,7)$ & $48(57,1)$ & $<0,005$ & $68(37,0)$ & $22(26,2)$ & 0,08 & $34(18,5)$ & $3(3,6)$ & $<0,005$ \\
\hline Cocaína & $123(66,8)$ & $39(46,4)$ & $<0,005$ & $71(38,6)$ & $20(23,8)$ & 0,02 & $35(19,0)$ & $3(4,0)$ & $<0,005$ \\
\hline Crack & $109(59,2)$ & $37(44,0)$ & 0,02 & $80(43,5)$ & $30(35,7)$ & 0,23 & $42(22,8)$ & $6(7,1)$ & $<0,005$ \\
\hline Inalantes ${ }^{1}$ & $79(42,9)$ & $32(38,1)$ & 0,52 & $6(3,3)$ & - & - & $1(0,5)$ & - & - \\
\hline Alucinógenos ${ }^{2}$ & $40(21,7)$ & $19(22,6)$ & 0,87 & $14(7,6)$ & $3(3,6)$ & $0,28^{*}$ & $2(1,1)$ & $2(2,4)$ & $0,42^{*}$ \\
\hline Outras & $20(10,9)$ & $9(10,7)$ & 0,97 & $3(1,6)$ & $1(1,2)$ & 1,00 & $1(0,6)$ & - & - \\
\hline
\end{tabular}

1Exemplos: cola, éter, lança-perfume, "Ioló", esmalte.

${ }^{2}$ Exemplos: LSD, êxtase, cogumelo, chás alucinógenos.

* Teste exato de Fisher. 
pesquisou a dependência do álcool por diferença de sexo e concluiu que a dependência de álcool é aproximadamente três vezes maior entre os homens que entre as mulheres ${ }^{3}$. No entanto, a comparação dos dados dos dois levantamentos tem demonstrado crescimento do uso de drogas entre as mulheres, com predominância do uso de medicamentos, como benzodiazepínicos e estimulantes ${ }^{2,3}$.

Entre as usuárias de drogas, o preconceito enfrentado se apresenta como um complicador da busca pelos serviços de atenção à dependência de drogas. O estigma social e sentimentos de culpa e de vergonha levam as mulheres a evitar expor seu problema e, consequentemente, buscar tratamento ${ }^{10,14}$. Um estudo sobre a adesão ao tratamento para a dependência de álcool e outras drogas demonstrou que, além da baixa procura pelo tratamento, o abandono também é mais prevalente entre mulheres ${ }^{7}$. No presente estudo, verificou-se menor proporção de mulheres no grupo de terceiro mês comparado ao grupo de início de tratamento (15,5\% e
$20,1 \%$, respectivamente), indicando prevalência ainda menor de mulheres em fases mais adiantadas de tratamento.

A baixa escolaridade encontrada entre os dependentes de álcool e outras drogas está em consonância com outros estudos da área ${ }^{6,12,15-18}$, os quais associam o uso de drogas a baixo desempenho escolar, além de maior índice de absenteísmo ${ }^{19}$. Aqui, no entanto, a baixa escolaridade também pode estar relacionada às características da clientela que busca os serviços assistenciais do SUS²0.

Em relação ao estado civil, entre o grupo que estava iniciando o tratamento, a maioria declarou-se não casada (solteira, divorciada ou viúva), enquanto no grupo no terceiro mês de tratamento, a maioria declarou-se casada, no entanto sem diferença significativa. Quando questionado com quem residia, a maior parcela dos entrevistados, em ambos os grupos, referiu morar com algum familiar ou amigo. Dessa forma, apesar de a maior parte dos indivíduos em tratamento nos CAPS-AD estar compreendida entre os es-

Tabela 3. Tempo de uso de drogas pelos dois grupos de pacientes estudados nos CAPS-AD em Curitiba, Paraná

\begin{tabular}{|c|c|c|c|c|c|c|c|c|}
\hline \multirow{2}{*}{\multicolumn{2}{|c|}{ Tipo de droga conforme grupo de tratamento }} & \multirow{3}{*}{$\mathrm{n}=184$} & \multicolumn{6}{|c|}{ Tempo de uso } \\
\hline & & & \multirow{2}{*}{$\begin{array}{c}\text { Uso experimental } \\
N(\%)\end{array}$} & \multirow{2}{*}{$\begin{array}{c}<1 \text { ano } \\
\mathrm{N}(\%)\end{array}$} & \multirow{2}{*}{$\begin{array}{c}\begin{array}{c}1 \mathrm{a}<2 \text { anos } \\
\mathrm{N}(\%)\end{array} \\
-\end{array}$} & \multirow{2}{*}{$\begin{array}{c}\begin{array}{c}2 \mathrm{a} 5 \text { anos } \\
\mathrm{N}(\%)\end{array} \\
10(5,4)\end{array}$} & \multirow{2}{*}{$\begin{array}{c}6 \mathrm{a} 10 \text { anos } \\
\mathbf{N}(\%)\end{array}$} & \multirow{2}{*}{$\begin{array}{c}>10 \text { anos } \\
\mathbf{N}(\%)\end{array}$} \\
\hline Álcool & Início & & & & & & & \\
\hline & $3^{\circ}$ mês & $\mathrm{n}=84$ & - & $1(1,2)$ & - & - & $5(6,0)$ & $78(92,9)$ \\
\hline & & $p\left(\chi^{2}\right)$ & - & $1,00^{*}$ & - & - & 0,36 & 0,05 \\
\hline \multirow[t]{3}{*}{ Tabaco } & Início & $n=152$ & - & $2(1,3)$ & $2(1,3)$ & $8(5,3)$ & $17(11,2)$ & $123(81)$ \\
\hline & $3^{\circ}$ mês & $n=76$ & $1(1,3)$ & $2(2,6)$ & $1(1,3)$ & $3(3,9)$ & $5(6,6)$ & $64(84,2)$ \\
\hline & & $p\left(\chi^{2}\right)$ & - & $0,6^{*}$ & $1,00^{*}$ & $0,76^{*}$ & 0,27 & 0,54 \\
\hline \multirow[t]{3}{*}{ Maconha } & Início & $n=143$ & $19(13,4)$ & $8(5,6)$ & $2(1,4)$ & $32(22,4)$ & $26(18,2)$ & $56(39,2)$ \\
\hline & $3^{0}$ mês & $\mathrm{n}=48$ & $7(14,6)$ & $6(12,5)$ & $2(4,2)$ & $8(16,7)$ & $7(14,6)$ & $18(37,5)$ \\
\hline & & $p\left(\chi^{2}\right)$ & 0,82 & 0,11 & 0,26 & 0,4 & 0,57 & 0,83 \\
\hline \multirow[t]{3}{*}{ Cocaína } & Início & $n=123$ & $14(11,4)$ & $19(15,4)$ & $7(5,7)$ & $34(27,6)$ & $11(8,9)$ & $38(30,9)$ \\
\hline & $3^{\circ}$ mês & $n=39$ & $9(23,1)$ & $4(10,3)$ & $2(5,1)$ & $4(10,3)$ & $8(20,5)$ & $12(30,8)$ \\
\hline & & $p\left(\chi^{2}\right)$ & 0,07 & $0,60^{*}$ & $1,00^{*}$ & $0,03^{*}$ & 0,05 & 0,99 \\
\hline \multirow[t]{3}{*}{ Crack } & Início & $\mathrm{n}=109$ & $10(9,2)$ & $15(13,8)$ & $6(5,5)$ & $18(16,5)$ & $19(17,4)$ & $41(37,6)$ \\
\hline & $3^{0}$ mês & $n=37$ & $4(10,8)$ & $3(8,1)$ & $2(5,4)$ & $9(24,3)$ & $8(21,6)$ & $11(29,7)$ \\
\hline & & $p\left(\chi^{2}\right)$ & 0,75 & $0,53^{*}$ & $1,00^{*}$ & 0,29 & 0,57 & 0,39 \\
\hline \multirow[t]{3}{*}{ Inalantes } & Início & $\mathrm{n}=79$ & $22(27,8)$ & $30(38,0)$ & $5(6,3)$ & $14(17,7)$ & $2(2,5)$ & $6(7,6)$ \\
\hline & $3^{\circ}$ mês & $n=32$ & $12(37,5)$ & $9(28,1)$ & $1(3,1)$ & $8(25,0)$ & $2(6,3)$ & - \\
\hline & & $p\left(\chi^{2}\right)$ & 0,32 & 0,33 & 0,67 & 0,38 & $0,58^{*}$ & - \\
\hline \multirow[t]{3}{*}{ Alucinógenos } & Início & $\mathrm{n}=40$ & $16(40,0)$ & $13(32,5)$ & $1(2,5)$ & $5(12,5)$ & $1(2,5)$ & $4(10,0)$ \\
\hline & $3^{0}$ mês & $\mathrm{n}=19$ & $7(36,8)$ & $7(36,8)$ & $2(10,5)$ & $2(10,5)$ & - & $1(5,3)$ \\
\hline & & $p\left(\chi^{2}\right)$ & 0,82 & 0,74 & $0,24^{*}$ & $1,00^{*}$ & - & $1,00^{*}$ \\
\hline \multirow[t]{3}{*}{ Outras } & Início & $n=20$ & $6(30,0)$ & $6(30,0)$ & $1(5,0)$ & $1(5,0)$ & - & $6(30,0)$ \\
\hline & $3^{0}$ mês & $\mathrm{n}=9$ & $3(33,3)$ & $2(22,2)$ & $1(11,1)$ & $3(33,3)$ & - & - \\
\hline & & $p\left(\chi^{2}\right)$ & $1,00^{*}$ & $1,00^{*}$ & $0,53^{*}$ & $0,08^{*}$ & - & - \\
\hline
\end{tabular}

${ }^{1}$ Uso experimental: poucos episódios de uso da droga (1 a 3 vezes).

* Teste exato de Fisher. 
tratos mais baixos de renda, os indivíduos que chegam ao tratamento nos CAPS-AD são, em sua maioria, pessoas que apresentam alguma rede de apoio social e condições mínimas de vida, como moradia. Os moradores de rua ou abrigos públicos corresponderam a apenas 1,5\% da amostra. Outro estudo também destaca que dependentes de drogas com maior comprometimento social parecem não chegar aos serviços de saúde e aponta para a necessidade de os municípios implementarem estratégias de facilitação do acesso, com maior envolvimento de agentes comunitários de saúde, com os Programas de Redução de Danos (PRD) ou com os Consultórios de Rua, ou ainda outras ações de aproximação entre comunidade e serviços ${ }^{13}$. Além disso, o modelo em que a porta de entrada para a tratamento é a Unidade de Saúde acaba por dificultar o acesso daqueles em maior vulnerabilidade social.

A principal diferença socioeconômica e demográfica entre os dois grupos estudados se deu em relação à faixa etá- ria. Indivíduos que estavam no terceiro mês de tratamento eram mais velhos que os iniciantes. Estudos que avaliaram a adesão ao tratamento de dependente de álcool ou outras drogas verificaram que o tempo de permanência nos centros de reabilitação é diretamente proporcional ao avanço da faixa etária ${ }^{6,721}$.

Tabela 5. Uso na vida de drogas nos dois grupos de pacientes estudados nos CAPS-AD, Curitiba, Paraná

\begin{tabular}{|c|c|c|c|}
\hline \multirow{2}{*}{ Usona vida } & $\begin{array}{l}\text { Início de tratamento } \\
\qquad(\mathrm{N}=184)\end{array}$ & Terceiro mês $(n=84)$ & \multirow{2}{*}{$p\left(\chi^{2}\right)$} \\
\hline & $N(\%)$ & $N(\%)$ & \\
\hline $\begin{array}{l}\text { Somente drogas } \\
\text { lícitas }^{1}\end{array}$ & $33(17,9)$ & $31(36,9)$ & $<0,001$ \\
\hline $\begin{array}{l}\text { Drogas lícitas e } \\
\text { ilícitas }^{2}\end{array}$ & $151(82,1)$ & $53(63,1)$ & \\
\hline
\end{tabular}

Tabela 4. Frequência semanal do uso de drogas nos dois grupos de pacientes estudados nos CAPS-AD em Curitiba, Paraná

\begin{tabular}{|c|c|c|c|c|c|c|c|}
\hline & & & & & ia de uso (dia. & & \\
\hline & forme grup & & Uso experimental' ${ }^{1}$ & $<1$ & $1 A 3$ & $3 A 6$ & Todos os dias \\
\hline Álcool & Início & $\mathrm{n}=184$ & - & $21(11,4)$ & $42(22,8)$ & $15(8,2)$ & $106(57,6)$ \\
\hline & $3^{\circ}$ mês & $\mathrm{n}=84$ & - & $9(10,7)$ & $9(10,7)$ & $3(3,6)$ & $63(75,0)$ \\
\hline & & $p\left(\chi^{2}\right)$ & - & 0,87 & 0,02 & $0,20^{*}$ & 0,006 \\
\hline Tabaco & Início & $n=152$ & - & $1(0,7)$ & $3(2,0)$ & $4(2,6)$ & $144(94,7)$ \\
\hline & $3^{\circ}$ mês & $n=76$ & $1(1,3)$ & - & - & $2(2,6)$ & $73(96,1)$ \\
\hline & & $p\left(\chi^{2}\right)$ & - & - & - & $1,00^{*}$ & $0,76^{*}$ \\
\hline Maconha & Início & $n=143$ & $19(13,3)$ & $22(15,4)$ & $28(19,6)$ & $1(0,7)$ & $73(51,0)$ \\
\hline & $3^{\circ}$ mês & $\mathrm{n}=48$ & $7(14,6)$ & $11(22,9)$ & $5(10,4)$ & $2(4,2)$ & $23(47,9)$ \\
\hline & & $p\left(\chi^{2}\right)$ & 0,82 & 0,23 & 0,15 & $0,16^{*}$ & 0,7 \\
\hline Cocaína & Início & $n=123$ & $14(11,4)$ & $26(21,1)$ & $48(39,0)$ & $6(4,9)$ & $29(23,6)$ \\
\hline & $3^{0}$ mês & $n=39$ & $9(23,1)$ & $4(10,3)$ & $13(33,3)$ & $2(5,1)$ & $11(28,2)$ \\
\hline & & $p\left(\chi^{2}\right)$ & 0,07 & $0,16^{*}$ & 0,52 & $1,00^{*}$ & 0,56 \\
\hline Crack & Início & $n=109$ & $10(9,2)$ & $16(14,7)$ & $19(17,4)$ & $12(11,0)$ & $52(47,7)$ \\
\hline & $3^{0}$ mês & $n=37$ & $4(10,8)$ & $3(8,1)$ & $4(10,8)$ & - & $26(70,3)$ \\
\hline & & $p\left(\chi^{2}\right)$ & $0,75^{*}$ & 0,30 & $0,43^{*}$ & - & 0,02 \\
\hline Inalantes & Início & $\mathrm{n}=79$ & $22(27,8)$ & $21(26,6)$ & $17(21,5)$ & $1(1,3)$ & $18(22,8)$ \\
\hline & $3^{0}$ mês & $n=32$ & $12(37,5)$ & $10(31,3)$ & $4(12,5)$ & - & $6(18,8)$ \\
\hline & & $p\left(\chi^{2}\right)$ & 0,32 & 0,62 & $0,42^{*}$ & - & 0,64 \\
\hline Alucinógenos & Início & $n=40$ & $16(40,0)$ & $12(30,0)$ & $10(25,0)$ & - & $2(5,0)$ \\
\hline & $3^{0}$ mês & $\mathrm{n}=19$ & $7(36,8)$ & $8(42,1)$ & $3(15,8)$ & - & $1(5,3)$ \\
\hline & & $p\left(\chi^{2}\right)$ & 0,82 & 0,36 & $0,52^{*}$ & - & $0,24^{*}$ \\
\hline Outras & Início & $n=20$ & $6(30,0)$ & $3(15,0)$ & $5(25,0)$ & $1(5,0)$ & $5(25,0)$ \\
\hline & $3^{0}$ mês & $\mathrm{n}=9$ & $3(33,3)$ & $1(33,3)$ & $2(22,2)$ & - & $1(11,1)$ \\
\hline & & $p\left(\chi^{2}\right)$ & $1,00^{*}$ & $1,00^{*}$ & $1,00^{*}$ & - & $0,64^{*}$ \\
\hline
\end{tabular}

${ }^{1}$ Uso experimental: poucos episódios de uso da droga (1 a 3 vezes).

* Teste exato de Fisher 
Em ambos os grupos, as drogas lícitas (álcool e tabaco) foram as mais utilizadas tanto na vida como no último ano e no último mês. Pesquisas em bases comunitárias revelam que o álcool e o tabaco são as drogas mais utilizadas pelo brasileiro, de forma geral, com aumento progressivo nos últimos anos 2,3,22,23. Além disso, também se pôde constatar que, entre aqueles que relataram fazer uso de álcool e tabaco, a maioria referiu fazer uso diário e há mais de 10 anos. Dados da pesquisa realizada em Teresina/PI, com 227 prontuários no CAPS-AD, também demonstrou alta frequência de uso de álcool entre os sujeitos estudados: 55\% da amostra relatava fazer uso diário de bebida alcoólica ${ }^{6}$.

O álcool é uma droga utilizada por muitos poliusuários como potencializadora ou inibidora dos efeitos de outras drogas. Sua ingestão, associada à cocaína, por exemplo, reforça os efeitos desta, tendendo o usuário a consumir doses crescentes tanto de uma quanto da outra quando o uso é feito de forma combinada. Já no consumo do crack, o álcool é utilizado posteriormente para diminuir a sensação de boca seca e amenizar outros efeitos indesejáveis ${ }^{24}$.

A alta porcentagem de indivíduos fazendo uso recente do tabaco encontrada neste estudo, $66,8 \%$ no grupo de início e $73,8 \%$ no grupo de terceiro mês, está em consonância com a literatura, que aponta que as taxas de fumantes entre indivíduos que buscaram tratamento para dependência de drogas são em média quatro vezes maiores do que na população geral ${ }^{25}$.

Uma característica marcante entre os pacientes em terceiro mês de tratamento foi que mais de um terço (37\%) nunca havia usado drogas ilícitas na vida. Isso sugere que grupos em estágios mais avançados de tratamento sejam compostos por usuários apenas de drogas lícitas (álcool e tabaco) e que o motivo da busca pelo tratamento seja pelo uso abusivo do álcool, uma vez que os CAPS-AD em questão não têm grupos de tratamento para dependentes exclusivos de tabaco. Também contribui para esse raciocínio o fato de que o grupo de terceiro mês de tratamento ingeria bebida alcoólica mais frequentemente do que o outro grupo.

No que concerne às drogas ilícitas, a mais utilizada na vida, em ambos os grupos, foi a maconha. O dado coincide com o obtido no Informe Mundial sobre Drogas - 2012 das Nações Unidas, que a destaca como a droga ilícita mais consumida no mundo ${ }^{26}$. Ela também é a droga ilícita mais utilizada pelo brasileiro ${ }^{3}$. Daqueles que afirmaram alguma vez ter feito uso de maconha na vida, a maior parte o fazia diariamente e há mais de 10 anos. Seu tempo estendido de utilização está relacionado ao fato de ela ser uma droga de iniciação dos poliusuários ${ }^{27,28}$. Além disso, o uso regular ou pesado associa-se a um risco aumentado de uso ou dependência de outras drogas ilícitas ${ }^{29}$.

O crack, por sua vez, foi a droga ilícita mais utilizada no último ano e no último mês nos dois grupos. O uso do crack vem apresentando aumento progressivo nos últimos anos ${ }^{2,3}$.
Segundo dados preliminares de uma pesquisa de âmbito nacional realizada em 2012, aproximadamente 2 milhões de brasileiros já usaram cocaína fumada (crack/merla e oxi) pelo menos uma vez na vida e 1 em cada 100 adultos usou crack no último ano, representando 1 milhão de pessoas ${ }^{30}$. Dessa forma, a dependência de crack já se configura como uma das principais causas de internamento para desintoxicação ${ }^{12,13,31}$.

Uma característica importante do usuário de crack é a utilização simultânea de múltiplas drogas. Assim, o indivíduo dificilmente consome a droga de forma isolada, associando-a principalmente ao álcool, maconha e tabaco, o que torna difícil a mensuração dos danos agregados a essas diversas associações ${ }^{13,32-34}$.

Outro achado relevante em relação ao crack foi o tempo prolongado de seu uso pelos pesquisados. Dos que afirmaram ter utilizado a droga, a maioria relatou consumo por mais de 10 anos. Em um estudo com uma coorte de usuários de crack por 12 anos também foi encontrada a manutenção do uso por tempo prolongado. Os autores apontam como causa dessa longevidade de uso as modificações que a droga tem sofrido ao longo dos anos. Com o objetivo de aumentar a lucratividade, são acrescidos diluentes, resultando em preparações mais "impuras" e, consequentemente, com menor potencial aditivo. Outra característica é o seu maior fracionamento, possibilitando menores preços e maior disseminação. Mais um fator apontado é que, apesar de o uso intensificado ainda ser o mais prevalente, alguns indivíduos conseguem desenvolver estratégias singulares de manutenção de padrões continuados de consumo em níveis moderados ${ }^{32}$.

Entre as drogas pesquisadas, os inalantes e alucinógenos foram as menos utilizadas. A maioria dos entrevistados afirmou ter apenas experimentado (fez uso em poucos episódios: uma a três vezes) ou utilizado por menos de um ano, e, entre aqueles que a utilizaram, a frequência semanal também foi baixa: a maior parte afirmou usar menos que uma vez por semana.

Os inalantes tiveram um uso na vida considerável: $42,9 \%$ no grupo de início de tratamento contra 38,1\% no grupo no terceiro mês de tratamento. No entanto, no grupo de início de tratamento, apenas 3,3\% relataram uso no último ano e 0,5\%, no último mês. Já no grupo de terceiro mês ninguém afirmou ter usado inalantes no ano e no último mês. Isso revela que, apesar de grande parcela de pacientes ter tido contato com essas drogas, elas, possivelmente, não são o principal motivador pela busca pelo tratamento. Os inalantes têm como característica serem drogas usadas por jovens no período de inicialização, sendo substituídos por drogas consideradas mais "pesadas" no decorrer do processo de abuso ${ }^{11,35}$

Os alucinógenos apresentaram um padrão de uso semelhante ao dos inalantes. Entretanto, os alucinógenos são descritos como drogas de uso esporádico, ligados a eventos especiais, como festas e rituais ${ }^{36}$. 
O baixo número de mulheres dependentes de drogas em tratamento nos CAPS-AD revela a importância de estratégias, no âmbito da atenção básica, que possam criar condições para que elas, primeiro, acessem o serviço e, posteriormente, apropriem-se do tratamento necessário, a fim de que possam encontrar o apoio que precisam para a sua recuperação. Nesse sentido, é preciso que o serviço leve em consideração as singularidades das mulheres, considerando os aspectos que permeiam o universo das usuárias de drogas. Da mesma forma, é necessária a atenção ao processo de tratamento dos pacientes mais jovens, por meio do desenvolvimento e execução de ações específicas que englobem a atração e manutenção no tratamento.

No que se refere aos padrões de uso de drogas, este estudo pôde demonstrar que a maioria dos pacientes dos CAPS-AD utiliza, ao longo da vida, vários tipos de substâncias psicoativas, algumas com característica de uso esporádico ou de experimentação, como os inalantes e alucinógenos, e outras com característica de uso prolongado, frequente e recente, como o álcool, o tabaco e o crack.

Pelo seu caráter de fácil acesso, o álcool e o tabaco, drogas lícitas, apresentam-se como sendo as mais consumidas pelos pacientes dos CAPS-AD. São diversos os agravos à saúde já relacionados a essas drogas. Nesse sentido, ressaltase a importância de ações de prevenção para abuso dessas drogas na população de forma geral, bem como estratégias específicas que atentem para a problemática de abuso de drogas lícitas pelos pacientes de CAPS-AD, mesmo entre aqueles cujo principal motivador da busca pelo tratamento foram as drogas ilícitas.

O fato de a amostra pretendida entre os pacientes de terceiro mês não ter sido alcançada pode ser um fator limitante desta pesquisa, ao passo que também reflete a dificuldade de indivíduos dependentes de drogas manterem-se em tratamento por períodos mais prolongados. O presente estudo, por características da sua própria natureza, não permitiu evidenciar os motivos que levaram às diferenças observadas, pois comparou dois grupos distintos em diferentes fases de tratamento. Outra limitação importante foi a não realização de diagnóstico de dependência para drogas específicas, mas apenas a caracterização em uso e não uso da drogas pesquisadas.

A principal contribuição desta pesquisa é a produção de informações sobre as características do público atendido pelos CAPS-AD, podendo subsidiar o desenvolvimento de estratégias efetivas para o tratamento e a detecção de grupos vulneráveis específicos. É também importante para a evidenciação de uma demanda reprimida, isto é, usuários que não buscam, ou, por motivos diversos, não chegam aos CAPS-AD. Dessa forma, novos estudos são necessários para compreender por que determinados grupos, como mulheres e moradores de rua, não acessam o CAPS-AD, e os motivos pelos quais grupos com determinadas características não se mantêm em tratamento.

\section{CONTRIBUIÇÕES INDIVIDUAIS}

Daniele do Rocio Ribeiro - Participou da concepção e desenho do estudo, da coleta de dados, da análise e interpretação dos dados, da elaboração do artigo e da aprovação da versão final.

Denise Siqueira de Carvalho - Participou da concepção e desenho do estudo, da análise e interpretação dos dados, da revisão crítica do conteúdo intelectual do artigo e da aprovação da versão final.

\section{CONFLITOS DE INTERESSE}

As autoras declaram não haver nenhum tipo de conflito de interesse.

\section{REFERÊNCIAS}

1. Escritório das Nações Unidas sobre Drogas e Crime. Relatório Mundial sobre as Drogas 2013.

2. Centro Brasileiro de Informações sobre Drogas Psicotrópicas (Cebrid). I levantamento domiciliar sobre o uso de drogas psicotrópicas no Brasil: estudo envolvendo as 107 maiores cidades do país: 2001. São Paulo; 2002.

3. Centro Brasileiro de Informações Sobre Drogas Psicotrópicas (Cebrid). II Levantamento domiciliar sobre o uso de drogas psicotrópicas no Brasil - 2005. São Paulo; 2005.

4. Brasil. Presidência da República. Secretaria Nacional de Políticas sobre Drogas. Relatório Brasileiro sobre Drogas. Brasília; 2009.

5. Brasil. Ministério da Saúde. Secretaria Executiva. Coordenação Nacional de DST/Aids. A Política do Ministério da Saúde para atenção integral a usuários de álcool e outras drogas. Braślia; 2003.

6. Monteiro CFS, Fé LCM, Moreira MAC, Albuquerque IEM, Silva MG, Passamani MC. Perfil sociodemográfico e adesão ao tratamento de dependentes de álcool em CAPS-AD do Piauí. Esc Anna Nery Rev Enferm. 2011;15(1):90-5.

7. Peixoto C, Henrique C, Prado DO, Rodrigues (P, Nelson J, Cheda D, et al. Impacto do perfil clínico e sociodemográfico na adesão ao tratamento de pacientes de um Centro de Atenção Psicossocial a Usuários de Álcool e Drogas (CAPS-AD). J Bras Psiquiatr. 2010;59(4):317-21.

8. Curitiba. Prefeitura Municipal de Curitiba. Protocolo Integrado de Saúde Mental em Curitiba. Curitiba; 2002.

9. Vargens RW, Cruz MS, Santos MA. Comparação entre usuários de crack e de outras drogas em serviço ambulatorial especializado de hospital universitário. Rev Latino-Am Enfermagem. 2011;19:804-12.

10. Araujo NB, Marcon SR, Silva NG, Oliveira JRT. Perfil clínico e sociodemográfico de adolescentes que permaneceram e não permaneceram no tratamento em um CAPS-AD de Cuiabá/MT. J Bras Psiquiatr. 2012;61(4):227-34.

11. Borini P, Guimarães RC, Borini SB. Usuários de drogas ilíitas internados em hospital psiquiátrico: padrões de uso e aspectos demográficos e epidemiológicos. J Bras Psiquiatr. 2003;52(3):171-9.

12. Capistrano FC, Ferreira ACZ, Silva TL, Kalinke LP, Maftum MA. Perfil sociodemográfico e clínico de dependentes químicos em tratamento: análise de prontuários. Esc Anna Nery Rev Enferm. 2013;17(2):234-41.

13. Horta RL, Horta BL, Rosset AP, Horta CL. Perfil dos usuários de crack que buscam atendimento em Centros de Atenção Psicossocial. Cad Saude Publica. 2011;27(11):2263-70.

14. Cesar BAL. Alcoolismo feminino: um estudo de suas peculiaridades - Resultados preliminares. J Bras Psiquiatr. 2006;55(2):208-11.

15. Formiga LT, Dumcke TS, Araujo RB. Comparing the profile of chemical dependents hospitalized at a chemical. Rev HCPA. 2006;29:120-6. 
16. Freire SD, Lucena P, Bortolini M, Feliz J, Moraes D. A classe econômica de usuários internados na cidade de Porto Alegre/Brasil. J Bras Psiquiatr. 2012;61(4):221-6.

17. Tavares BF, Béria JU, Lima MS. Prevalência do uso de drogas e desempenho escolar entre adolescentes. Rev Saude Publica. 2001;35(2):150-8.

18. Queiroz S, Scivoletto S, Silva MMS, Strassman PG, Andrade AG, Gattaz WF. Uso de drogas entre estudantes de uma escola pública de São Paulo. Rev Psiq Clín. 2001;28(4):176-82.

19. Galduróz JCF, Noto AR, Fonseca AM, Carlini EA. Centro Brasileiro de Informações sobre Drogas Psicotrópicas (Cebrid). V levantamento nacional sobre o consumo de drogas psicotrópicas entre estudantes do ensino fundamental e médio da rede pública de ensino nas 27 capitais brasileiras 2004. São Paulo; 2004.

20. Ribeiro MCSA, Barata RB, Almeida MF, Silva ZP. Perfil sociodemográfico e padrão de utilização de serviç̧os de saúde para usuários e não-usuários do SUS - PNAD 2003. Ciênc Saúde Coletiva. 2006;11(4):1011-22.

21. Burns L, Randall D, Hall WD, Law M, Butler T, Bell J, et al. Opioid agonist pharmacotherapy in New South Wales from 1985 to 2006: patient characteristics and patterns and predictors of treatment retention. Addiction. 2009;104(8):1363-72.

22. Bastos Fl, Bertoni N, Hacker MA. Consumo de álcool e drogas: principais achados de pesquisa de âmbito nacional, Brasil 2005. Rev Saude Publica. 2008;42(Suppl 1).

23. Fonseca AM, Carlos J, Galduróz F, Noto AR. 0 uso de drogas no Brasil: comparação de dois levantamentos domiciliares: 2001 e 2004. Ciênc Saúde Coletiva. 2004;15:663-70.

24. Gossop M, Manning V, Ridge G. Concurrent use and order of use of cocaine and alcohol: behavioural differences between users of crack cocaine and cocaine powder. Addiction. 2006;101(9):1292-8.

25. Williams JM, Ziedonis $D$. Addressing tobacco among individuals with a mental illness or an addiction. Addict Behav. 2004;29(6):1067-83.
26. Nações Unidas. Informe mundial sobre las drogas. Nova York; 2012.

27. Agrawal A, Neale MC, Prescott CA, Kendler KS. A twin study of early cannabis use and subsequent use and abuse/dependence of other illicit drugs. Psychol Med. 2004;34(7):122737.

28. Morral AR, McCaffrey DF, Paddock SM. Reassessing the marijuana gateway effect. Addiction. 2002:97(12):1493-504

29. Fergusson DM, Boden JM, Horwood LJ. Cannabis use and other illicit drug use: testing the cannabis gateway hypothesis. Addiction. 2006;101(4):556-69.

30. Instituto Nacional de Políticas Públicas do Álcool e Outras Drogas (INPAD). Universidade Federal de São Paulo. II Levantamento Nacional de Álcool e Drogas (II LENAD) - dados preliminares. São Paulo; 2013

31. Filho OFF, Turchi MD, Laranjeira R, Castelo A. Perfil sociodemográfico e de padrões de uso entre dependentes de cocaína hospitalizados. Rev Saude Publica. 2003;37(6):751-9.

32. Dias AC, Araújo MR, Laranjeira R. Evolução do consumo de crack em coorte com histórico de tratamento. Rev Saude Publica. 2011;45(5):938-48.

33. Rodrigues DS, Backes DS, Freitas HMB, Zamberlan C, Gelhen MH, Colomé JS. Conhecimentos produzidos acerca do crack: uma incursão nas dissertações e teses brasileiras. Ciênc Saúde Coletiva. 2012;17(5):1247-58.

34. Guimarães CF, Santos DVV, Freitas RC, Araujo RB. Perfil do usuário de crack e fatores relacionados à criminalidade em unidade de internação para desintoxicação no Hospital Psiquiátrico São Pedro de Porto Alegre (RS). Rev Psiquiatr Rio Gd Sul. 2008;30(2):101-8.

35. Filho AJA, Ferreira MA, Gomes MLB, Silva RC, Santos TCF. 0 adolescente e as drogas: consequências para a saúde. Esc Anna Nery Rev Enferm. 2007;11(4):605-10.

36. de Almeida SP, Silva MT. Ecstasy (MDMA): effects and patterns of use reported by users in São Paulo. Rev Bras Psiquiatr. 2003;25(1):11-7. 